\title{
GR3027 reversal of neurosteroid-induced, GABA-A receptor-mediated inhibition of human brain function: an allopregnanolone challenge study
}

\author{
Maja Johansson ${ }^{1,2}$ (D) Maria Månsson ${ }^{1} \cdot$ Lars-Eric Lins $^{1} \cdot$ Bruce Scharschmidt $^{1} \cdot$ Magnus Doverskog $^{1}$ • \\ Torbjörn Bäckström ${ }^{1,2}$
}

Received: 20 December 2017 / Accepted: 15 February 2018 / Published online: 28 February 2018

(C) The Author(s) 2018. This article is an open access publication

\begin{abstract}
Rationale GR3027 is a novel small molecule GABA-A receptor-modulating steroid antagonist, which in non-clinical studies has shown promise for treatment of human disorders due to allosteric over-activation of GABA-A receptors by neurosteroids, such as allopregnanolone. We here studied its safety, pharmacokinetics, and ability to inhibit allopregnanolone effects in humans.

Methods Safety and pharmacokinetics were studied in healthy adult males receiving ascending single or multiple oral GR3027 vs. placebo. GR3027-mediated reversal of allopregnanolone effect on maximal saccadic eye velocity (SEV), and self-rated somnolence was studied in a double-blind, placebo-controlled, three-part cross-over study in which 3 or $30 \mathrm{mg}$ oral GR3027 preceded $0.05 \mathrm{mg} / \mathrm{kg}$ of i.v. allopregnanolone.

Results GR3027 was well tolerated, adverse events were generally mild and transient, and no dose-limiting toxicity or grade 3 adverse events were observed up to the highest single $(200 \mathrm{mg}$ ) or multiple ( $100 \mathrm{mg}$ every $12 \mathrm{~h}$ for 5 days) doses. The maximum concentration $\left(C_{\max }\right)$ and systemic exposure (area under the plasma concentration-time curve from dose extrapolated to infinity $\left[\mathrm{AUC}_{0-\infty}\right]$ and/or AUC during the dosing interval $\left[\mathrm{AUC}_{\tau}\right]$ ) varied linearly with dose; with dose-dependent accumulation ratios of 1.3-1.6. Allopregnanolone decreased SEV and induced somnolence in most, but not all subjects. By predefined analyses, $30 \mathrm{mg}$ GR3027 significantly inhibited allopregnanolone-induced decrease in SEV $(p=0.03) ; 3$ and $30 \mathrm{mg}$ GR3027 non-significantly inhibited allopregnanolone-induced sedation. By post hoc analyses restricted to subjects with allopregnanolone-induced changes and the time period over which they occurred, GR3027 dose dependently inhibited allopregnanolone-induced decrease in SEV ( $p=0.04$ at $30 \mathrm{mg}$, non-significant at $3 \mathrm{mg}$ ) and allopregnanolone-induced sedation ( $p=0.01 / 0.05$ at 3/30 $\mathrm{mg}$ doses).

Conclusion Oral GR3027 mitigates inhibition of brain function induced by allopregnanolone at doses which are clinically well tolerated and associated with linear pharmacokinetics.
\end{abstract}

Keywords GR3027 · Allopregnanolone $\cdot$ Clinical trial $\cdot$ Saccadic eye movement $\cdot$ Sedation

Electronic supplementary material The online version of this article (https://doi.org/10.1007/s00213-018-4864-1) contains supplementary material, which is available to authorized users.

Maja Johansson

Maja.Johansson@Umecrine.se

1 Umecrine Cognition AB, Karolinska Institutet Science Park, Fogdevreten 2, SE-171 65 Solna, Sweden

2 Department of Clinical Sciences, Obstetrics and Gynecology, Umeå University, SE-901 87 Umeå, Sweden

\section{Introduction}

The GABA system is the major inhibitory neurotransmitter system in the brain. It regulates diverse CNS functions including learning, memory, sedation, and sleep and is a validated pharmacological target (Olsen and Sieghart 2008). While a variety of GABA-A agonist drugs have been approved, attempts to develop GABAA antagonists have been hindered by their propensity to induce seizures. Indeed, pentylenetetrazol, a GABA-A antagonist, is commonly used as an experimental CNS convulsant (Adeoluwa et al. 2016).

GABA-A receptors are ionotropic heteropentameric assemblies derived from a family of 19 members $(6 \alpha, 3 \beta, 3 \gamma$, 
$\delta, \varepsilon, \theta, \pi, 3 \rho)$, with 20 distinct configurations described (Olsen and Sieghart 2008). Their functional characteristics vary depending on receptor subtype and localization within the brain and within the neuron. For example, extrasynaptic $\alpha 5$ subunit-containing GABA-A receptors have a wellestablished role in the hippocampus, a brain area involved in learning and memory, and represent an attractive target for memory-enhancing drugs with antagonizing properties (Mohler and Rudolph 2017). By contrast, activation of $\alpha 1$ subunit-containing GABA-A receptors, which are more dispersed and most often located within the synapse, induces sedation and sleep (Mohler 2002). The GABA system is also involved in sleep regulation (Saper et al. 2010; Scammell et al. 2017). Most sedative/hypnotics used for insomnia target the GABA-A receptor (Winsky-Sommerer 2009), and activation of GABA-A receptors in the posterior hypothalamus promotes sleep and contributes to both homeostatic and circadian sleep regulation (Volgin et al. 2014).

In addition to GABA, endogenous $3 \alpha, 5 \alpha$ - and $3 \alpha, 5 \beta$-reduced metabolites of progesterone such as allopregnanolone ( $3 \alpha$-hydroxy-5 $\alpha$-pregnane-20-one) and THDOC $(3 \alpha, 5 \alpha$ tetrahydrodeoxycorticosterone), commonly referred to as neurosteroids, act as strong positive allosteric modulators of the GABA-mediated activation of both synaptic and extrasynaptic GABA-A receptors (Majewska et al. 1986), thus enhancing both phasic and tonic inhibition. Allopregnanolone is a potent anesthetic and induces an inhibitory GABAergic tone in the CNS that alters the sleep/wake cycle and impairs memory and learning. Inhibition of the effects of neurosteroids on the GABA-A receptor holds promise as a therapeutic approach to revere GABAergic-mediated inhibition of CNS function without the analeptic effects associated with GABA-A receptor antagonists (Johansson et al. 2016). GR3027 is a novel investigational drug that has been shown in vitro to selectively antagonize the positive modulation of $\alpha 1$ - and $\alpha 5$-subunit-containing GABA-A receptors by neurosteroids, i.e., potentially influencing both phasic and tonic inhibition, without antagonizing the effects of GABA and in vivo to restore spatial learning and motor coordination in two animal models of hepatic encephalopathy (Johansson et al. 2015; Johansson et al. 2016). In the present studies, we have examined its safety, tolerability, and pharmacokinetics in humans during single and multiple ascending dose (SAD and MAD) studies in healthy adults as well as its ability to enter the CNS and exhibit GABA-A receptor-modulating steroid antagonist properties in the context of an allopregnanolone challenge using maximal saccadic eye velocity (SEV) and sedation/somnolence as pharmacodynamic markers.

Saccadic eye velocity is used to assess the effects of GABA-A receptor agonists such as allopregnanolone or benzodiazepines (Ball et al. 1991; Timby et al. 2006). The saccade is the fast movement of the eye when the gaze is shifted to a new peripheral visual stimulus. When a new visual target is presented, the gaze, after a latency period, moves towards the new target (Carpenter 2000). The maximal speed reached during the saccade, i.e., the SEV, is dependent on the angle between the targets, is regulated by the activity of the GABAA receptor, and is not under conscious control (Ball et al. 1991). SEV is, therefore, a sensitive and quantifiable measure of GABA-A receptor modulation by compounds like allopregnanolone or benzodiazepines (Ball et al. 1991; Carpenter 2000; Moller et al. 2016; Timby et al. 2006). A GABA-A-mediated decrease in SEV, for example by allopregnanolone, is generally accompanied by increased sedation/somnolence (Timby et al. 2006; van Broekhoven et al. 2007).

The present studies demonstrate that GR3027, a novel oral GABA-A receptor-modulating steroid antagonist, enters the brain and inhibits the effect of the neurosteroid allopregnanolone in humans at doses which are well within the range that exhibits excellent tolerability and pharmacokinetics. The studies build on prior work exploring the ability of isoallopregnanolone (an endogenous neurosteroid antagonist) to antagonize the effects of allopregnanolone on SEV and sedation in animals and humans (Backstrom et al. 2005; Bengtsson et al. 2015).

\section{Methods}

\section{Study design}

The first in human GR3027 SAD study (part 1 of EudraCT 2015-004911-19) and the 5-day MAD study (EudraCT 2016003651-30) were prospective, randomized, double-blind and placebo-controlled and designed to assess the safety, tolerability, and pharmacokinetic characteristics of single and multiple ascending oral doses of GR3027 in healthy male volunteers. GR3027 (SAD 1, 3, 10, 30, 100, and $200 \mathrm{mg}$; MAD $50 \mathrm{mg} /$ day, 50 or $100 \mathrm{mg}$ twice daily (BID)) was administered orally in the morning or every $12 \mathrm{~h}$.

The antagonist study (part 2 of EudraCT 2015-004911-19) was randomized and placebo-controlled and had a doubleblind three-part cross-over design, with at least 1 week between test occasions. In the morning, study subjects orally received placebo (A), $3 \mathrm{mg}$ GR3027 (B), or $30 \mathrm{mg}$ GR3027 (C) followed 90 min later by an intravenous (i.v.) allopregnanolone challenge and underwent repeated measurement of SEV and sedation/somnolence before administrations and for $3 \mathrm{~h}$ thereafter. Subjects were randomized concerning the order of the oral treatments, so that three subjects followed the same sequence, i.e., $\mathrm{ABC}, \mathrm{ACB}, \mathrm{BAC}, \mathrm{BCA}, \mathrm{CAB}$, and CBA, respectively.

The studies were performed in accordance with ethical principles that have their origin in the Declaration of Helsinki and are consistent with ICH/Good Clinical Practice 
(GCP) E6(R1), European Union (EU) Clinical Trials Directive, and applicable local regulatory requirements. The study was approved by the Independent Ethics Committee in Uppsala, Sweden, and by the Swedish Medical Product Agency, and was performed at Clinical Trial Consultants AB (CTC) in Uppsala, Sweden.

\section{Subjects}

Ninety (90) eligible healthy consenting male subjects (age 1850 years) were included in the studies and randomized to treatment (GR3027 or placebo 6:2 in SAD and MAD protocols) or treatment sequence in the antagonist study (6 groups of 3 individuals). Attending subjects were recruited from the CTC database of persons interested in participating in clinical trials or by advertisements. All randomized subjects completed the SAD and MAD studies, while 17 subjects completed all study visits in the antagonist study and one subject was withdrawn when presenting at the clinic for the second treatment period. Withdrawal was due to moderate sadness (depressed mode), sleepiness (somnolence), and mild vertigo that were assessed as not related to study treatment.

Exclusion criteria included the following: present (assessed by medical examination, ECG, and laboratory values at screening) or history of any clinically significant disease or disorder, including anxiety (GAD-7 questionnaire); depression (PHQ-9 questionnaire and the The Columbia-Suicide Severity Rating Scale), which could either put the subject at risk or influence the results; use of any prescribed or nonprescribed medication including antacids, analgesics, herbal remedies, vitamins, and minerals within 2 weeks prior to study visits, except the occasional intake of paracetamol and nasal decongestants without cortisone or antihistamine for a maximum of 10 days; a history of alcohol abuse; positive screen for drugs of abuse or alcohol; and present or historic use of anabolic steroids. During the study, consumption of alcohol, coffee (SAD and antagonist study), xanthine- or taurinecontaining products/beverages, and grapefruit or grapefruitcontaining products was not allowed, nor was smoking or use of nicotine-containing products.

\section{Trial compounds}

GR3027 (Umecrine Cognition AB, Sweden) was administered orally in gelatin capsules, 1 or $10 \mathrm{mg}$ per capsule (RISE, Bioscience and Materials - Surface, Process and Formulation, Södertälje, Sweden). Placebo capsules contained excipients only and were of identical appearance as the GR3027 capsules.

The allopregnanolone (Umecrine AB, Sweden) formulation was made by Cobra Biologics Matfors, Sweden, in a concentration of $0.13 \mathrm{mg} / \mathrm{mL}$ allopregnanolone dissolved in an albumin solution $(200 \mathrm{mg} / \mathrm{mL})$.

\section{Experimental protocols}

The screening visit included a physical examination, historical and baseline medical characteristics, and inclusion/exclusion criteria as well as signing of informed consent. Subjects were confined to the clinic from the evening before dosing until $24 \mathrm{~h}$ (SAD and antagonist study) or $48 \mathrm{~h}$ (MAD) post last dose. A follow-up visit was performed 5-10 days, or 21 days (MAD), after the last dose of GR3027.

Study days with PK sampling or SEV testing were preceded by an 8-h overnight fasting period before administration of the oral placebo or GR3027; food intake was at the earliest $2 \mathrm{~h}$ after dose. In the antagonist study, allopregnanolone $(0.05 \mathrm{mg}$ / $\mathrm{kg}$ body weight) was administered as an i.v. infusion $(2 \mathrm{mg} /$ min over $\sim 1-2 \mathrm{~min}$ ) $90 \mathrm{~min}$ after placebo/GR3027.

Vital signs, including body temperature, blood pressure, heart rate, and clinical chemistry (e.g., alkaline phosphatase, alanine aminotransferase, aspartate aminotransferase, bilirubin, creatinine), were followed during the experimental period.

\section{Saccadic eye measurements}

For calculation of SEV, saccadic eye measurements (SEM) were performed at pre-oral GR3027 dosing, preallopregnanolone dosing (-10 $\mathrm{min})$, and 10, 20, 40, 60, 80, $100,120,140,160$, and $180 \mathrm{~min}$ after allopregnanolone.

A Saccadometer Plus device (Ober Consulting Sp. z o.o, Poland) using direct infrared photo-oculography was used with visual stimuli 20 degrees apart horizontally. Measurements for an individual subject were performed at approximately the same time at each of the three study visits. At each time point, data were collected from 30 saccades. Not all saccades were evaluable. The saccade data used for analysis fulfilled the following prespecified criteria: were not rejected by the automatic validation, amplitude between 15 and 25 degrees, duration below $150 \mathrm{~ms}$, and latency above $50 \mathrm{~ms}$. Data from time points with less than 15 evaluable saccades were regarded as missing. For data analysis, the median SEV at each time point was used. The change in SEV induced by the allopregnanolone injection $(\triangle \mathrm{SEV})$ was calculated with the SEV measure $10 \mathrm{~min}$ before allopregnanolone as baseline value. $\triangle \mathrm{SEV}$ was used as SEV varies greatly between individuals but has a high intraindividual stability, both within testing sessions and between testing days (Mercer et al. 1990; Wilson et al. 1993). For calculation of $\triangle \mathrm{SEV}$ area under the curve $\left(\mathrm{AUC}_{\mathrm{t}} \triangle \mathrm{SEV}\right)$, missing data points were imputed with the use of present data points just before and just after the missing point.

The response to allopregnanolone alone was evaluated at the study visit when oral placebo was given before the allopregnanolone injection. Allopregnanolone-responding 
subjects were defined as those showing a decrease in SEV during at least two succeeding measurements.

\section{Sedation}

Before each SEM time point, sedation was assessed by the subject using a visual analogue scale (VAS) with the lower end representing "Absence of sleepiness" $(0 \mathrm{~mm})$ and the upper end indicating "Falling asleep" $(100 \mathrm{~mm})$ and the value scored recorded. Data were treated similarly to SEV data, as described above; there were no missing data.

The response to allopregnanolone alone was evaluated at the study visit when it was preceded by oral placebo. Allopregnanolone-responding subjects were defined as those scoring an increase in sedation lasting for at least two measures in a row, with an increase of at least $10 \mathrm{~mm}$ in the peak.

\section{Blood sampling}

Samples were collected pre-dose and at 20, 40, 60, and $90 \mathrm{~min}$ and 2, 3, 4, 6, 9, 12, 18, and $24 \mathrm{~h}$ following dosing in the SAD protocol and the MAD $50 \mathrm{mg}$ /day group and after last dose in all MAD groups. With BID dosing, the 18- and 24-h time points were omitted after the first dose. In the SAD study, 48-h samples were obtained after the higher single doses and in the MAD study after the last dose. In the MAD study, predose samples were collected all days.

In the antagonist study, blood samples for analysis of serum levels of allopregnanolone $(-10,10,20,40,60,120$, and $180 \mathrm{~min})$ and plasma levels of GR3027 (-10, 60, 120, $180 \mathrm{~min}$ ) were collected through an indwelling venous catheter.

Collected samples were stored at minus $70{ }^{\circ} \mathrm{C}$ and shipped on dry ice for analyses.

\section{Analyses of GR3027 and allopregnanolone concentrations}

The plasma concentration of GR3027 and serum concentration of allopregnanolone were quantified by UPLC/MS/MS Waters Acquity, C18 columns, and Waters XEVO-TQ-S triple quadrupole mass spectrometers at the National Veterinary Institute (SVA; Uppsala, Sweden; GR3027) and at Admescope Oy (Oulu, Finland, allopregnanolone). As internal standards, D4-GR3027 and D5-allopregnanolone were used, respectively. For analysis of allopregnanolone, oxime derivatization was used to increase sensitivity (KeskiRahkonen et al. 2011). Standard and QC samples were prepared into human blank plasma/serum. The detection limit for GR3027 was $1 \mathrm{ng} / \mathrm{mL}$ and for allopregnanolone $0.05 \mathrm{ng} / \mathrm{mL}$ $(0.15 \mathrm{nmol} / \mathrm{L})$.

\section{Pharmacokinetic analyses}

The following pharmacokinetic parameters were evaluated using non-compartmental analysis and the software Phoenix WinNonlin ${ }^{\circledR}$ version 6.3 or later (Pharsight Corporation, USA): maximal concentration $\left(C_{\max }\right)$, time point for $C_{\max }$ $\left(T_{\max }\right)$, terminal half-life $\left(T_{1 / 2}\right)$, area under the curve extrapolated to infinity $\left(\mathrm{AUC}_{0 \rightarrow \infty}\right)$, area under the curve during dosing interval $\left(\mathrm{AUC}_{\tau}\right)$, and total apparent body clearance following extravascular administration (CL/F). Dose proportionality was calculated for the SAD study based on $\mathrm{AUC}_{0 \rightarrow \infty}$ and $C_{\max }$, and in the MAD study on steady-state $\mathrm{AUC}_{\tau}$. In the MAD study, the accumulation ratio $\left(R_{\mathrm{A}, \mathrm{AUC}}\right)$ between the first and last doses was calculated as (steady-state $\mathrm{AUC}_{\tau}$ firstdose $\mathrm{AUC}_{\tau}$ ).

\section{Statistical analyses}

Statistical calculations were with SAS® (Version 9.4, SAS Institute Inc., Cary, NC, USA) and IBM SPSS statistics (Version 24, IBM).

Treatment differences based on $\mathrm{AUC}_{\mathrm{t}} \Delta \mathrm{SEV}$ and $\mathrm{AUC}_{\mathrm{t}}$ $\triangle$ VAS (prespecified, assuming the duration of the allopregnanolone effect to be $180 \mathrm{~min}$ ), the same parameters divided by the actual allopregnanolone AUC in each subject (prespecified), allopregnanolone exposures (AUC allopregnanolone) with different oral pretreatment (prespecified), and SEV and VAS measures before allopregnanolone were analyzed using Wilcoxon's signedrank test. For non-related samples, i.e., allopregnanolone concentration vs. body mass index (BMI), the Mann-Whitney $U$ test was used. Estimates of the treatment effect were also performed with a prespecified mixed model repeated measure (MMRM) analysis with $\triangle \mathrm{SEV}$ or $\triangle$ VAS as dependent variables and treatment, visit, and time point as fixed effect independent variables. The sample size of 18 for the allopregnanolone challenge study was based on power calculations indicated that 14 and 17 evaluable subjects were needed for 80 and $90 \%$ power, respectively, to detect a true difference in a two-sided test at the 5\% significance level.

During statistical analysis of allopregnanolone-responding subjects, one subject showing an atypical response to allopregnanolone was excluded, i.e., for this subject, SEV continuously decreased and sedation continuously increased during the study visit.

\section{Results}

\section{Subject recruitment and disposition}

Of the 48 and 24 subjects who met all entry criteria and were enrolled in the SAD and MAD protocols, respectively, all 
completed the study and were included in the final analyses. Among the 18 subjects enrolled to the antagonist study, 1 subject was excluded because of an adverse event (AE) judged unrelated to treatment, while 17 completed the protocol and were included in the prespecified analyses (Supplementary Fig. 1). Post hoc analyses which excluded patients who exhibited either no effect or an atypical effect of allopregnanolone included 11 of the 17 subjects for SEV $(65 \%)$ and 8 of 17 subjects for sedation (47\%).

\section{Safety and tolerability}

Single- and multiple-dose oral administration of GR3027 was generally well tolerated by the subjects, no serious adverse events (SAEs) occurred, and all AEs were transient and classified as mild (grade 1) or moderate (grade 2). No clinically significant abnormal physical examination findings or clinical chemistry values were reported at any of the time points assessed.

\section{SAD}

Treatment-emergent AEs possibly related to treatment were reported by four subjects (11\%) receiving GR3027, while no possibly or probably related AEs were reported by subjects that received placebo $(0 \%)$. No increased frequency or intensity of events was seen by increasing GR3027 dose. The possibly related AEs reported were headache $(n=3)$, pruritus $(n=1)$, and rash $(n=1)$.

\section{MAD}

Thirteen treatment-emergent AEs possibly or probably related to treatment were reported by eight subjects receiving GR3027 (44\%): three subjects (50\%) treated with $50 \mathrm{mg}$ GR3027 once daily, one subject (17\%) treated with $50 \mathrm{mg}$ GR3027 twice daily (BID), and four subjects (67\%) treated with $100 \mathrm{mg}$ GR3027 BID. Five treatment-emergent AEs possibly or probably related to treatment were reported by two subjects (33.3\%) that received placebo. Most of the symptoms were related to the gastrointestinal tract ( $50 \mathrm{mg}, 2$ AEs (2 subjects); $50 \mathrm{mg}$ BID, 0 AE; 100 mg BID, 5 AEs (3 subjects); placebo, 2 AEs (1 subject)), all were of short duration (minutes to few hours) and most were graded as mild, with one moderate. There were also reports of fatigue $(50 \mathrm{mg} ; n=1)$, thirst (50 mg BID; $n=1$ ), dizziness (100 mg BID; 1 mention each by 2 subjects), and headache (100 mg BID; 1 mention each by 2 subjects).

\section{Agonist study}

Four subjects experienced treatmen-emergent AEs possibly or probably related to treatment, i.e., allopregnanolone or
GR3027 (24\%). While receiving GR3027 plus allopregnanolone, headache $(n=1)$ and abdominal pain $(n=$ 1) were reported, both during treatment with $3 \mathrm{mg}$ GR3027. When treatment was with placebo plus allopregnanolone, headache was reported by three subjects.

\section{Pharmacokinetics}

After single dosing with GR3027, the plasma concentration increased proportionally to the increase in dose with proportionality constants $\beta$ of 1.01 for $C_{\max }$ and of 1.12 for $\mathrm{AUC}_{0-\infty}$ (Fig. 1a and supplementary Table 1). Dosing for 5 days twice daily resulted in a proportional increase in steady-state plasma GR3027 with 1.9 times increase in $\mathrm{AUC}_{\tau}$ when the dose was doubled from 50 to $100 \mathrm{mg}$ BID, and with mean ( \pm $\mathrm{SD})$ accumulation ratios of $1.3 \pm 0.1$ (50 $\mathrm{mg}$ once daily), $1.5 \pm 0.3$ (50 mg BID), and $1.6 \pm 0.4$ (100 mg BID) (Fig. 1b, c and supplementary Table 1).

\section{GR3027 antagonism of allopregnanolone-induced decreased SEV: prespecified analyses}

The allopregnanolone-induced change in SEV (placebo pretreatment; $\triangle \mathrm{SEV}$ ) analyzed as area under the curve (AUC) was $-4025 \pm 2215(\min \times \mathrm{deg} / \mathrm{s})$; a similar $\mathrm{AUC}_{\mathrm{t}}$ $\triangle \mathrm{SEV}$ of $-4042 \pm 1654(\mathrm{~min} \times \mathrm{deg} / \mathrm{s})$ was found for $3 \mathrm{mg}$ GR3027 pretreatment, while $\mathrm{AUC}_{\mathrm{t}} \Delta \mathrm{SEV}$ for pretreatment with $30 \mathrm{mg}$ GR3027 was $-1494 \pm 1840$ $(\mathrm{min} \times \mathrm{deg} / \mathrm{s})$. Thus, $30 \mathrm{mg}$ GR3027 significantly antagonized the allopregnanolone-induced change in SEV $(p=0.030$, Wilcoxon's signed-rank test, Fig. 2(a)). There was no antagonism by the $3 \mathrm{mg}$ GR3027 dose $(p=0.89)$.

The statistical significance for $30 \mathrm{mg}$ GR3027 was also present in relation to the actual allopregnanolone exposure ( $\mathrm{AUC}_{\mathrm{t}} \triangle \mathrm{SEV} / \mathrm{AUC}_{\mathrm{t}}$ allopregnanolone). For allopregnanolone $-2.61 \pm 1.51(\mathrm{~min} \times \mathrm{deg} / \mathrm{s} / \mathrm{min} \times \mathrm{ng} / \mathrm{mL})$ and $-0.94 \pm 1.23(\mathrm{~min} \times \mathrm{deg} / \mathrm{s} / \mathrm{min} \times \mathrm{ng} / \mathrm{mL})$ with $30 \mathrm{mg}$ GR3027 ( $p=0.041)$.

Allopregnanolone decreased SEV with a maximal effect of $-42.9 \pm 9.1 \mathrm{deg} / \mathrm{s}$ at the first studied time point $10 \mathrm{~min}$ after the administration (Fig. 2(b), placebo treatment). The SEV reduction gradually diminished until $80 \mathrm{~min}$ after allopregnanolone but did not return to baseline. When study subjects received GR3027 before the allopregnanolone administration, there was also a decrease in SEV at the early time points with maximal decrease 20 min after the allopregnanolone administration, i.e., $-25.3 \pm 12.6 \mathrm{deg} / \mathrm{s}$ $(3 \mathrm{mg})$ and $-16.7 \pm 10.2 \mathrm{deg} / \mathrm{s}(30 \mathrm{mg})$. With $30 \mathrm{mg}$ GR3027, SEV was back to baseline at the end of the study period. 


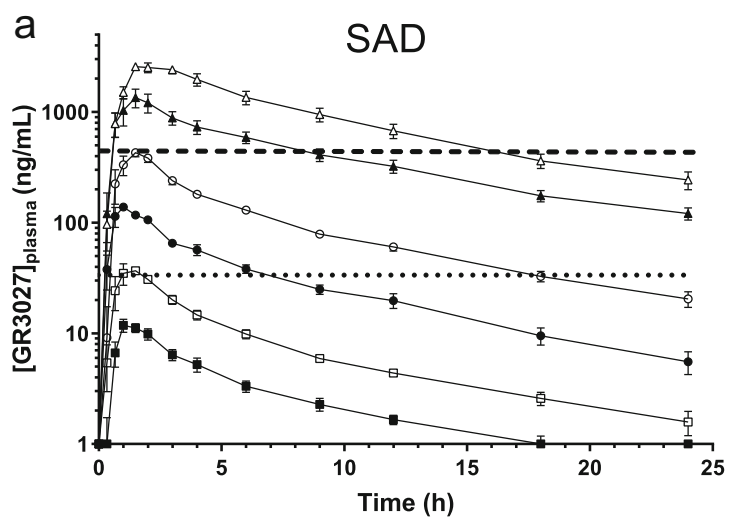

$$
\begin{aligned}
& \rightarrow 200 \mathrm{mg} \\
& -100 \mathrm{mg} \\
& -30 \mathrm{mg} \\
& \rightarrow 10 \mathrm{mg} \\
& \square-3 \mathrm{mg} \\
& -1 \mathrm{mg}
\end{aligned}
$$
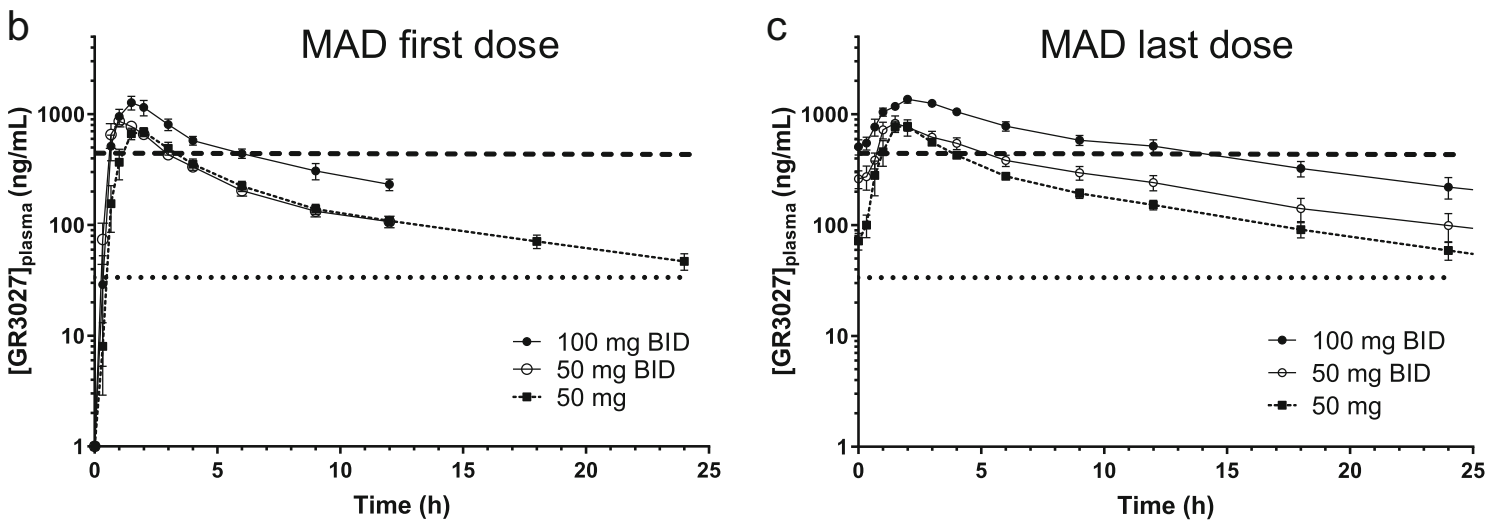

Fig. 1 GR3027 plasma concentrations in the SAD and MAD studies. The three panels depict GR3027 concentrations in healthy adult males during $\mathrm{SAD}$ (a) and during days 1 (b) and 5 (c) of MAD, $n=6$ for each dose.

Horizontal lines represent the peak concentrations of GR3027 following the 3 and $30 \mathrm{mg}$ doses used in the $3 \mathrm{~h}$ allopregnanolone challenge study. Values below $1 \mathrm{ng} / \mathrm{mL}$ are shown as $1 \mathrm{ng} / \mathrm{mL}$ (mean $\pm \mathrm{SEM}$ )

Statistical analyses over the whole study period (up to 180 min after allopregnanolone) with mixed model repeated

measures of $\triangle \mathrm{SEV}$ was inconclusive due to considerable variability that was not explained by the hypothesized model.

Fig. 2 Effect of oral GR3027 on the allopregnanolone-induced change in maximal saccadic eye velocity and sedation, prespecified analyses with Wilcoxon's signed-rank test including all evaluable data $(n=17$ subjects) for the whole study period, up to $180 \mathrm{~min}$ after the allopregnanolone injection. (a) Area under curve for $\triangle \mathrm{SEV}$. (b) $\triangle \mathrm{SEV}$ over time (mean $\pm \mathrm{SEM}$ ). (c) Area under curve for sedation. (d) Change in sedation over time (mean \pm SEM). Note that $30 \mathrm{mg}$ GR3027 significantly antagonized the allopregnanoloneinduced change in $\operatorname{SEV}(p=$ 0.030 ), whereas $3 \mathrm{mg}$ GR3027 did not $(p=0.89)$
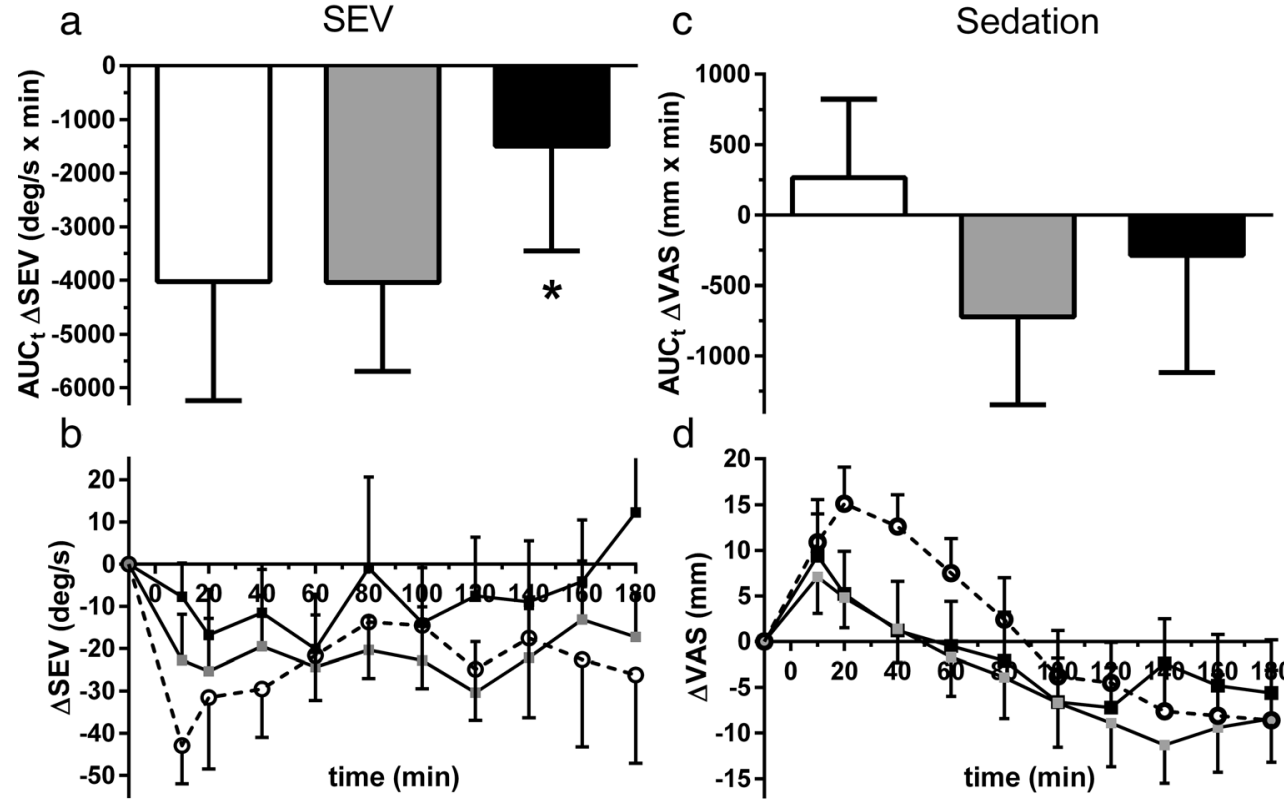

d

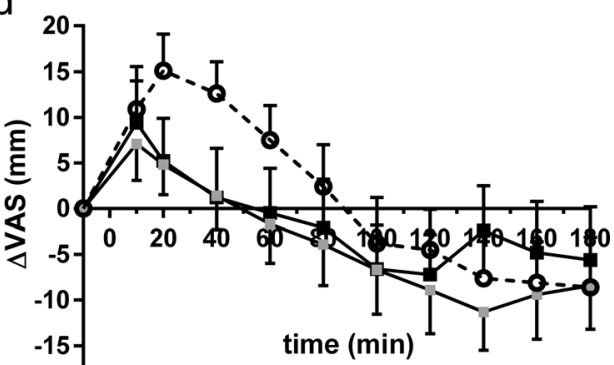

-๑. Allopregnanolone + placebo
-- Allopregnanolone + GR3027 $3 \mathrm{mg}$
- Allopregnanolone + GR3027 $30 \mathrm{mg}$ 
Post hoc analyses of GR3027 antagonism of change in SEV among allopregnanolone-responding subjects during the responsive period

When subjects responding to the allopregnanolone injection with a decrease in SEV were studied separately, it was obvious that the SEV decrease induced by allopregnanolone lasted only approximately $80 \mathrm{~min}$ after the injection (Fig. 3(b)) rather than the $180 \mathrm{~min}$ assumed in the prespecified analyses. Statistical analysis of $\mathrm{AUC}_{\mathrm{t}} \Delta \mathrm{SEV}$ from the responsive period (Fig. 3(a)) showed that $30 \mathrm{mg}$ GR3027 significantly inhibited the allopregnanolone-induced decrease in $\operatorname{SEV}(p=0.04)$, while the diminution of the allopregnanolone effect by $3 \mathrm{mg}$ GR3027 was non-significant $(p=0.29) . \mathrm{AUC}_{\mathrm{t}} \Delta \mathrm{SEV}$ for allopregnanolone was $-3018 \pm 656 \mathrm{~min} \times \mathrm{deg} / \mathrm{s}$, while $\mathrm{AUC}_{\mathrm{t}} \Delta \mathrm{SEV}$ with $3 \mathrm{mg}$ GR3027 was $-1592 \pm 924 \mathrm{~min} \times$ $\mathrm{deg} / \mathrm{s}$ and with $30 \mathrm{mg}$ GR3027-909 $\pm 798 \mathrm{~min} \times \mathrm{deg} / \mathrm{s}$.

In this study population, allopregnanolone maximally decreased SEV by $-62.5 \pm 13.0 \mathrm{deg} / \mathrm{s}$ at $20 \mathrm{~min}$ after allopregnanolone injection (Fig. 3b). GR3027 antagonized the allopregnanolone-induced changes so that the SEV measures at the same time point were $-29.5 \pm 15.9 \mathrm{deg} / \mathrm{s}(3 \mathrm{mg}$ GR3027) and $-18.0 \pm 14.2 \mathrm{deg} / \mathrm{s}$ (30 mg GR3027).

\section{Absence of effect of GR3027 alone on SEV}

On the three study occasions, SEV measured just prior to the oral capsules was $519 \pm 19.6 \mathrm{deg} / \mathrm{s}$ (placebo), $520 \pm 21.9 \mathrm{deg} / \mathrm{s}$ (3 mg GR3027), and 519 $\pm 25.0 \mathrm{deg} / \mathrm{s}$ (30 mg GR3027), and 90 min after the oral capsules but just prior to allopregnanolone was $532 \pm 19.8 \mathrm{deg} / \mathrm{s}$ (placebo), $519 \pm$ $15.7 \mathrm{deg} / \mathrm{s}(3 \mathrm{mg}$ GR3027), and $508 \pm 20.4 \mathrm{deg} / \mathrm{s}(30 \mathrm{mg}$ GR3027). All measurements were similar and there was no evidence of change in SEV by GR3027 per se.

\section{GR3027 antagonism of allopregnanolone-induced sedation: prespecified analyses}

There were no statistically significant changes in sedation by GR3027 when all subjects were included in the analysis of the whole study period (Fig. 2(c and d)).

Allopregnanolone increased the self-rated sedation (Fig. 2(d), placebo treatment), with the highest sedative score shown at the period 20 to $40 \mathrm{~min}$ after the injection of + $15.1 \pm 5.5$ and $+12.6 \pm 5.0 \mathrm{~mm}$, at 20 and $40 \mathrm{~min}$ respectively. Also, the sedative effect lasted for $80 \mathrm{~min}$ while thereafter the study subjects experienced even less sedation than before allopregnanolone. GR3027 (3 and $30 \mathrm{mg}$ ) appeared to decrease sedation induced by allopregnanolone; however, this effect was non-significant by the predefined statistical analyses. With the mixed model repeated measure analysis of change in sedation over time, convergence criteria for the hypothesized model were met but there was limited variability in the data ( $3 \mathrm{mg} p=0.67,30 \mathrm{mg} p=0.90)$. No post hoc analysis was performed to explore additional interactions or parameters that could explain changes in sedation. Analysis of the area under the curve for change in sedation $\left(\mathrm{AUC}_{\mathrm{t}} \Delta \mathrm{VAS}\right.$, Fig. 3a) or $\mathrm{AUC}_{\mathrm{t}} \Delta \mathrm{VAS}$ divided by $\mathrm{AUC}_{\mathrm{t}}$ allopregnanolone concentration showed neither any significant inhibition by
Fig. 3 Effect of oral GR3027 on the allopregnanolone-induced change in maximal saccadic eye velocity and sedation in allopregnanolone-responding subjects $(n=11$ for SEV and $n=8$ for sedation), post hoc analyses during the responsive period, up to $80 \mathrm{~min}$ after the allopregnanolone injection. (a) Area under curve for $\triangle \mathrm{SEV}$. (b) $\triangle \mathrm{SEV}$ over time (mean $\pm \mathrm{SEM}$ ). 30 mg GR3027 significantly inhibited the allopregnanoloneinduced decrease in SEV $(p=$ 0.04 ), whereas the diminution of the allopregnanolone effect by 3 mg GR3027 was nonsignificant $(p=0.29)$. (c) Area under curve for sedation. (d) Change in sedation over time (mean \pm SEM). Both 3 ( $p=$ $0.012)$ and $30 \mathrm{mg}(p=0.05)$ GR3027 significantly inhibited the sedative effect
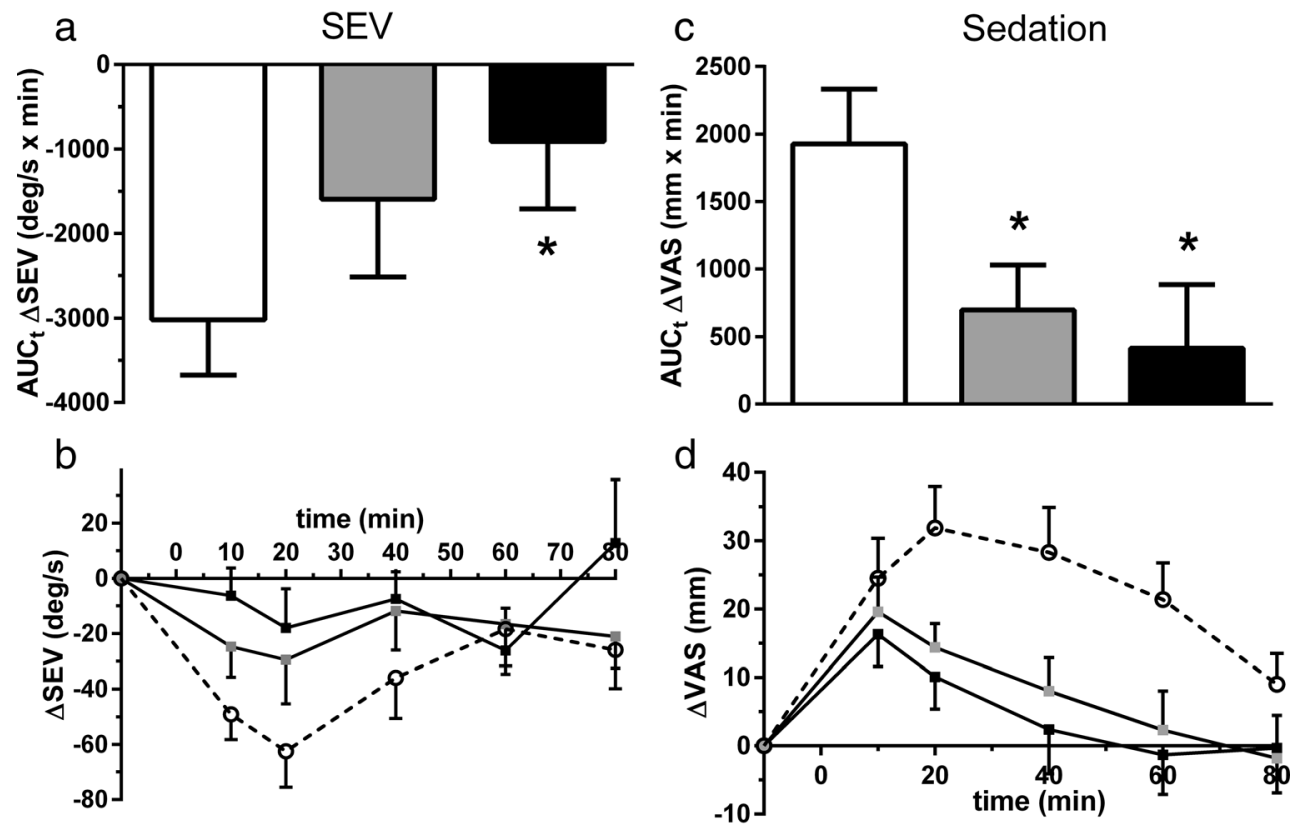

-๑. Allopregnanolone + placebo

-- Allopregnanolone + GR3027 $3 \mathrm{mg}$

- Allopregnanolone + GR3027 30 mg 
GR3027 ( $3 \mathrm{mg}, p=0.31$ and 0.28 , respectively; $30 \mathrm{mg}, p=$ 0.61 and 0.55 , respectively).

\section{Post hoc analyses of GR3027 antagonism of sedation among allopregnanolone-responding subjects during the responsive period}

For subjects responding to the allopregnanolone injection with sedation, the statistical analysis showed that both 3 and $30 \mathrm{mg}$ GR3027 significantly inhibited the sedative effect $(p=0.012$ and $p=0.05$, respectively). The mean AUCs of sedation $\left(\mathrm{AUC}_{\mathrm{t}} \triangle \mathrm{VAS}\right)$ after the different treatments were $1928 \pm$ $404 \mathrm{~min} \times \mathrm{mm}$ (placebo), $698 \pm 332 \mathrm{~min} \times \min (3 \mathrm{mg}$ GR3027), and $416 \pm 469 \mathrm{~min} \times \min (30 \mathrm{mg} \mathrm{GR} 3027)$ (Fig. $3(\mathrm{c}))$.

After allopregnanolone, this study population scored a maximal increase of $31.9 \pm 6.5 \mathrm{~mm}$ on the VAS scale for measure of sedation, with an increase above $20 \mathrm{~mm}$ for the period 10 to $60 \mathrm{~min}$ after allopregnanolone (Fig. 3(d)). With GR3027 pretreatment, the largest sedative increases were $19.6 \pm 5.4$ and $16.3 \pm 5.0 \mathrm{~mm}$, with 3 and $30 \mathrm{mg}$ GR3027 respectively, and sedation thereafter quickly diminished.

\section{GR3027 by itself did not affect sedation}

On the three study occasions, sedation levels were assessed just before oral GR3027: $24 \pm 4.6 \mathrm{~mm}$ (placebo), $30 \pm 4.3 \mathrm{~mm}$ (3 mg GR3027), and $26 \pm 4.2 \mathrm{~mm}$ (30 mg GR3027), as well as $90 \mathrm{~min}$ after the oral treatment but prior to allopregnanolone: $27 \pm 4.8 \mathrm{~mm}$ (placebo, $p=0.033$ ), $30 \pm 4.7 \mathrm{~mm} \mathrm{(3 \textrm {mg }}$ GR3027, $p=0.11$ ), and $32 \pm 6.3 \mathrm{~mm}$ (30 mg GR3027, $p=$ $0.06)$. Sedation levels before and after GR 3027 were very similar and not-statistically different; a significant $\sim 3$-mm increase in sedation was seen by placebo treatment.

\section{Exposures to GR3027 and allopregnanolone}

The GR3027 plasma concentration after $3 \mathrm{mg}$ oral GR3027 was $102 \pm 14 \mathrm{nM}$ just before the injection of allopregnanolone. The GR3027 concentration was then relatively stable during the period with high allopregnanolone exposure, with a $20 \%$ decrease during that hour. With $30 \mathrm{mg}$ oral GR3027, the plasma concentration was $1294 \pm 106 \mathrm{nM}$ at the time for the allopregnanolone injection and with a similar kinetic profile as for the low dose (supplementary Fig. 2).

At the three study occasions, the serum allopregnanolone concentration curves were very similar (supplementary Fig. 2) with the highest serum level at the first study time point after the i.v. injection, 74-82 nM. The allopregnanolone concentrations thereafter quickly decreased and were almost back to baseline $3 \mathrm{~h}$ after the injection. There were no significant differences between the allopregnanolone exposures during the three study occasions ( $p=0.16$, comparisons of $\mathrm{AUC}_{\mathrm{t}}$ allopregnanolone).

Subjects with a BMI of 25 or higher $(n=7)$ had a significantly higher allopregnanolone concentration at the first studied time point, $88 \pm 2.8 \mathrm{nM}$, compared to those with $\mathrm{BMI}<25$ $(n=10), 66 \pm 2.7 \mathrm{nM}(p=0.001$, Mann-Whitney $U$ test, mean BMI 24, range $20-28 \mathrm{~kg} / \mathrm{m}^{2}$ ). However, there was no significant difference between the BMI groups when serum allopregnanolone over the whole study period was analyzed ( $p=0.143$, Mann-Whitney $U$ test).

\section{Discussion}

The present findings demonstrate that orally administered GR3027 is well tolerated in humans. AEs were generally mild and neither SAEs nor dose-limiting toxicity was observed up to the highest doses administered to healthy adult males in the single (200 mg) or multiple (100 mg every $12 \mathrm{~h}$ for 5 days) ascending dose protocols. At all doses studied, GR3027 exhibited linear PK, i.e., both $C_{\max }$ and systemic exposure assessed as $\mathrm{AUC}_{0-\infty}(\mathrm{SAD})$ or $\mathrm{AUC}_{\tau}$ (steady-state in MAD) were linearly related to oral dose. During steady-state dosing, accumulation ratios were dose dependent and ranged from 1.3 to 1.6 .

Importantly, the results of the challenge study further indicate that GR3027 enters the brain and antagonizes the effects of allopregnanolone at the GABA-A receptor. By prespecified analysis, GR3027 at $30 \mathrm{mg}$, but not $3 \mathrm{mg}$, significantly inhibited the effects of allopregnanolone on SEV, whereas neither doses had a significant effect on sedation. However, based on post hoc analyses restricted to just those subjects who exhibited an allopregnanolone-induced change in SEV or self-rated sedation and to just the time interval during which these effects were observed, orally administered GR3027 produced apparent dose-dependent inhibition of the effects of allopregnanolone. SEV was significantly inhibited by $30 \mathrm{mg}$ GR3027 with an intermediate, but non-significant, inhibition at $3 \mathrm{mg}$. GR3027 also significantly reversed allopregnanolone-induced sedation at both the 3 and $30 \mathrm{mg}$ doses, again in apparent dose-dependent fashion.

The prespecified analyses in the present study were based on prior studies of allopregnanolone administration to healthy adult females (Bengtsson et al. 2015; Hedstrom et al. 2015; Timby et al. 2016). While the effects of the allopregnanolone challenge observed in the present study in healthy males, including the maximal change in both SEV and sedation, are generally similar to those previously described in healthy adult females (Bengtsson et al. 2015; Hedstrom et al. 2015; Moller et al. 2016; Timby et al. 2016), interesting differences were observed. For example, only $50-70 \%$ of the healthy adult male subjects exhibited sedation and/or SEV inhibition in the present study vs. over $90 \%$ of healthy adult females 
given the same milligram per kilogram dose of allopregnanolone (unpublished data). Moreover, among those males who did respond to allopregnanolone, the effect lasted for a shorter period of time, i.e., $\sim 80 \mathrm{~min}$ for both SEV and sedation, vs. 120 to more than $180 \mathrm{~min}$ in females given similar doses (Hedstrom et al. 2015; Moller et al. 2016; Timby et al. 2016). Although decreased sensitivity to allopregnanolone-induced changes in SEV has been observed in overweight females (Hedstrom et al. 2015), response among males to allopregnanolone in the present study appeared unrelated to either BMI or serum allopregnanolone levels.

We are aware of one prior study (van Broekhoven et al. 2007) in which both men and women received i.v. allopregnanolone that suggested the presence of genderrelated differences. At the highest dose $(0.045 \mathrm{mg} / \mathrm{kg})$, males exhibited a smaller SEV response than females while no gender difference in SEV response was reported at lower doses. By contrast, males exhibited more sedation than females in response to low doses of allopregnanolone, but also seemed to recover more quickly than females. In the von Broekhoven study (van Broekhoven et al. 2007), serum allopregnanolone concentrations increased significantly more in males vs. females. These findings in aggregate suggest that while the overall response to an allopregnanolone challenge is similar among healthy adult males and females, there are likely subtle gender-related differences in allopregnanolone uptake and/or disposition and/or GABA-A receptor sensitivity to allopregnanolone that account for the differences in the response to allopregnanolone exhibited by males vs. females (Backstrom et al. 2013; Hedstrom et al. 2015; Timby et al. 2016).

The favorable safety and pharmacokinetic findings as well as the results of the allopregnanolone challenge study suggest that GR3027 represents a promising new treatment for human disorders attributable to the CNS effects of neurosteroids. Hepatic encephalopathy (HE), a neurological disorder associated with cirrhosis and portal-systemic shunting in which elevated brain levels of allopregnanolone have been reported (Ahboucha et al. 2005; Ahboucha et al. 2006), is one example. The manifestations of HE comprise a wide spectrum that ranges from subtly impaired covert neuropsychiatric abnormalities detectable only by standardized testing to clinically overt abnormalities that include altered motor function, sleep disturbances with changes in the sleep-wake cycle, daytime drowsiness, slurred speech, inappropriate behaviour, impaired memory impairment, shortened attention span, altered mental status with disorientation, coma, and even death (Bajaj et al. 2011; Montagnese et al. 2015; Vilstrup et al. 2014). Indeed, GR3027 has shown promise in two different animal models of $\mathrm{HE}$, including rats with portacaval shunts and rats with chronic hyperammonemia, both of which are associated with increased brain concentrations of neurosteroids (Ahboucha et al. 2008; Cauli et al. 2009). In both animal models, GR3027 restored motor coordination, spatial learning, and spatial memory and partially restored the abnormal circadian rhythm in portacaval shunt rats (Johansson et al. 2015).

The apparently greater effect of GR3027 on reversing allopregnanolone's effect on sedation vs. SEV in the present study in adult males is an interesting finding reminiscent of prior findings in the portacaval shunt rat model of HE (Johansson et al. 2015), in which a lower subcutaneous dose of GR3027 restored motor coordination compared to the higher dose needed to restore learning in the radial maze. One potential explanation for this differing dose-dependency is that different areas of the brain with differing GABA-A receptor subtypes are responsible for different neurological functions, i.e., SEV is specifically controlled by GABA-A receptors in the superior colliculus and in cerebellum (Hikosaka and Wurtz 1983; Kojima et al. 2010), while spatial learning is mainly modulated in the hippocampus (Moser et al. 2017). It is also possible that uptake and/or disposal of allopregnanolone or GR3027 differs among differing brain areas (Bixo et al. 1997; Johansson et al. 2015; Wang et al. 1995).

The findings of the challenge study extend the earlier findings in animal models. While the CNS concentrations of allopregnanolone resulting from the i.v. challenge cannot be directly measured, currently available data suggest that they are pathophysiologically relevant to those in human disease states and that GR3027 antagonizes the effects of allopregnanolone at such concentrations. Serum allopregnanolone concentrations measured in adult males 10 min after injection averaged $\sim 80 \mathrm{nM}$, considerably higher than concentrations of $6-20 \mathrm{nmol} / \mathrm{kg}$ in the brain of cirrhotic patients who died in hepatic coma (Ahboucha et al. 2005; Ahboucha et al. 2006). Moreover, animal studies indicate that the concentration vs. time profile for GR3027 (unpublished data) and allopregnanolone (Johansson et al. 2002) is very similar in brain and plasma. The results imply that GR3027 administered at doses as low as $30 \mathrm{mg}$, well within the range that is well tolerated in adult males, can reverse the effects of neurosteroids even at concentrations higher than those described in HE patients. Importantly, GR3027 by itself had no effect on either SEV or sedation. This is consistent with in vitro findings and its mechanism of action whereby it would be expected to selectively reverse only neurosteroid-mediated modulation of GABA-A receptors.

Several recent observations also implicate neurosteroidinduced allosteric activation of CNS GABA-A receptors in the excessive daytime sleepiness (EDS) associated with disorders such as idiopathic hypersomnia (Billiard and Sonka 2016). These include the identification of a population of GABAergic neurons in the ventral lateral hypothalamus that help mediate human wakefulness (Venner et al. 2016), the identification of a putative GABA-A agonist in the CSF of 
patients with primary hypersomnia (Rye et al. 2012), and the clinical observation that the GABA-A antagonist flumazenil can promote wakefulness in patients with EDS (Trotti et al. 2016). Indeed, the clinical manifestations of HE and hypersomnolence disorders exhibit similarities, and HE has been considered a sleepiness disorder (Montagnese et al. 2015).

In summary, these findings show that GR3027 administered orally to humans antagonizes the effects of the neurosteroid allopregnanolone at the GABA-A receptor in the brain at doses which are well tolerated and associated with linear pharmacokinetics. This suggests that GR3027 may also be able to normalize GABAergic neurotransmission and represents a promising new therapeutic for treatment of HE, disorders associated with hypersomnolence or other disorders attributable to neurosteroid-mediated allosteric overactivation of GABA-A receptors.

Acknowledgements The authors would like to acknowledge the staff at Clinical Trial Consultants AB (CTC) in Uppsala, Sweden, where the studies described in this manuscript were performed, and at PCG Clinical Services, Uppsala, Sweden, that conducted data management, clinical monitoring, and prespecified biostatistics.

Funding information Umecrine Cognition $\mathrm{AB}$, Sweden, was the sponsor of the study.

\section{Compliance with ethical standards}

Conflict of interest MJ, MM, LEL, BS, and MD are/were employees of and/or consultants to Umecrine Cognition; TB and BS are shareholders and members of the Board of Directors of Umecrine Cognition.

Open Access This article is distributed under the terms of the Creative Commons Attribution 4.0 International License (http:// creativecommons.org/licenses/by/4.0/), which permits unrestricted use, distribution, and reproduction in any medium, provided you give appropriate credit to the original author(s) and the source, provide a link to the Creative Commons license, and indicate if changes were made.

\section{References}

Adeoluwa OA, Aderibigbe AO, Agu GO (2016) Pharmacological evaluation of central nervous system effects of ethanol leaf extract of Olax subscorpioidea in experimental animals. Drug Res (Stuttg) 66: 203-210. https://doi.org/10.1055/s-0035-1564137

Ahboucha S, Jiang W, Chatauret N, Mamer O, Baker GB, Butterworth RF (2008) Indomethacin improves locomotor deficit and reduces brain concentrations of neuroinhibitory steroids in rats following portacaval anastomosis. Neurogastroenterol Motil 20:949-957. https://doi.org/10.1111/j.1365-2982.2008.01132.x

Ahboucha S, Layrargues GP, Mamer O, Butterworth RF (2005) Increased brain concentrations of a neuroinhibitory steroid in human hepatic encephalopathy. Ann Neurol 58:169-170. https://doi.org/10.1002/ ana.20534

Ahboucha S, Pomier-Layrargues G, Mamer O, Butterworth RF (2006) Increased levels of pregnenolone and its neuroactive metabolite allopregnanolone in autopsied brain tissue from cirrhotic patients who died in hepatic coma. Neurochem Int 49:372-378. https://doi. org/10.1016/j.neuint.2006.02.002

Backstrom T, Bixo M, Nyberg S, Savic I (2013) Increased neurosteroid sensitivity - an explanation to symptoms associated with chronic work related stress in women? Psychoneuroendocrinology 38: 1078-1089. https://doi.org/10.1016/j.psyneuen.2012.10.014

Backstrom T, Wahlstrom G, Wahlstrom K, Zhu D, Wang MD (2005) Isoallopregnanolone; an antagonist to the anaesthetic effect of allopregnanolone in male rats. Eur J Pharmacol 512:15-21. https:// doi.org/10.1016/j.ejphar.2005.01.049

Bajaj JS, Cordoba J, Mullen KD, Amodio P, Shawcross DL, Butterworth RF, Morgan MY, International Society for Hepatic Encephalopathy and Nitrogen Metabolism (ISHEN) (2011) Review article: the design of clinical trials in hepatic encephalopathy - an International Society for Hepatic Encephalopathy and Nitrogen Metabolism (ISHEN) consensus statement. Aliment Pharmacol Ther 33:739747. https://doi.org/10.1111/j.1365-2036.2011.04590.x

Ball DM, Glue P, Wilson S, Nutt DJ (1991) Pharmacology of saccadic eye movements in man. 1. Effects of the benzodiazepine receptor ligands midazolam and flumazenil. Psychopharmacology (Berl) 105:361-367

Bengtsson SK, Nyberg S, Hedstrom H, Zingmark E, Jonsson B, Backstrom T, Bixo M (2015) Isoallopregnanolone antagonize allopregnanolone-induced effects on saccadic eye velocity and self-reported sedation in humans. Psychoneuroendocrinology 52: 22-31. https://doi.org/10.1016/j.psyneuen.2014.10.025

Billiard M, Sonka K (2016) Idiopathic hypersomnia. Sleep Med Rev 29: 23-33. https://doi.org/10.1016/j.smrv.2015.08.007

Bixo M, Andersson A, Winblad B, Purdy RH, Backstrom T (1997) Progesterone, 5alpha-pregnane-3,20-dione and 3alpha-hydroxy-5alpha-pregnane-20-one in specific regions of the human female brain in different endocrine states. Brain Res 764:173-178

Carpenter RH (2000) The neural control of looking. Curr Biol 10:R291R293

Cauli O, Mansouri MT, Agusti A, Felipo V (2009) Hyperammonemia increases GABAergic tone in the cerebellum but decreases it in the rat cortex. Gastroenterology 136:1359-1367, e1351-1352. https:// doi.org/10.1053/j.gastro.2008.12.057

Hedstrom H, Backstrom T, Bixo M, Nyberg S, Wang M, Gideonsson I, Turkmen S (2015) Women with polycystic ovary syndrome have elevated serum concentrations of and altered $\mathrm{GABA}(\mathrm{A})$ receptor sensitivity to allopregnanolone. Clin Endocrinol 83:643-650. https://oi.org/10.1111/cen.12809

Hikosaka O, Wurtz RH (1983) Effects on eye movements of a GABA agonist and antagonist injected into monkey superior colliculus. Brain Res 272:368-372

Johansson IM, Birzniece V, Lindblad C, Olsson T, Backstrom T (2002) Allopregnanolone inhibits learning in the Morris water maze. Brain Res 934:125-131

Johansson M, Agusti A, Llansola M, Montoliu C, Strömberg J, Malinina E, Ragagnin G, Doverskog M, Bäckström T, Felipo V (2015) GR3027 antagonizes GABAA receptor-potentiating neurosteroids and restores spatial learning and motor coordination in rats with chronic hyperammonemia and hepatic encephalopathy. Am J Physiol Gastrointest Liver Physiol 309:G400-G409. https://doi. org/10.1152/ajpgi.00073.2015

Johansson M, Stromberg J, Ragagnin G, Doverskog M, Backstrom T (2016) GABAA receptor modulating steroid antagonists (GAMSA) are functional in vivo. J Steroid Biochem Mol Biol 160:98-105. https://doi.org/10.1016/j.jsbmb.2015.10.019

Keski-Rahkonen P, Huhtinen K, Poutanen M, Auriola S (2011) Fast and sensitive liquid chromatography-mass spectrometry assay for seven androgenic and progestagenic steroids in human serum. J Steroid Biochem Mol Biol 127:396-404. https://doi.org/10.1016/j.jsbmb. 2011.06.006 
Kojima Y, Soetedjo R, Fuchs AF (2010) Effects of GABA agonist and antagonist injections into the oculomotor vermis on horizontal saccades. Brain Res 1366:93-100. https://doi.org/10.1016/j.brainres. 2010.10.027

Majewska MD, Harrison NL, Schwartz RD, Barker JL, Paul SM (1986) Steroid hormone metabolites are barbiturate-like modulators of the GABA receptor. Science 232:1004-1007

Mercer AJ, Marshall RW, Richens A (1990) Saccadic eye movements as a method of psychometric assesment. In: Klepper ID, Saunders LD, Rosen M (eds) Ambulatory anaesthesia and sedation. Blackwell, Oxford, pp 88-105

Mohler H (2002) Pathophysiological aspects of diversity in neuronal inhibition: a new benzodiazepine pharmacology. Dialogues Clin Neurosci 4:261-269

Mohler H, Rudolph U (2017) Disinhibition, an emerging pharmacology of learning and memory F1000Res 6 doi:https://oi.org/10.12688/ f1000research.9947.1

Moller AT, Backstrom T, Nyberg S, Sondergaard HP, Helstrom L (2016) Women with PTSD have a changed sensitivity to GABA-A receptor active substances. Psychopharmacology 233:2025-2033. https:// doi.org/10.1007/s00213-014-3776-y

Montagnese S, Turco M, Amodio P (2015) Hepatic encephalopathy and sleepiness: an interesting connection? J Clin Exp Hepatol 5:S49 S53. https://doi.org/10.1016/j.jceh.2014.06.006

Moser EI, Moser MB, McNaughton BL (2017) Spatial representation in the hippocampal formation: a history. Nat Neurosci 20:1448-1464. https://doi.org/10.1038/nn.4653

Olsen RW, Sieghart W (2008) International Union of Pharmacology. LXX. Subtypes of gamma-aminobutyric acid(A) receptors: classification on the basis of subunit composition, pharmacology, and function. Update. Pharmacol Rev 60:243-260. https://doi.org/10.1124/ pr.108.00505

Rye DB, Bliwise DL, Parker K, Trotti LM, Saini P, Fairley J, Freeman A, Garcia PS, Owens MJ, Ritchie JC, Jenkins A (2012) Modulation of vigilance in the primary hypersomnias by endogenous enhancement of GABAA receptors. Sci Transl Med 4:161ra151. https://doi.org/ 10.1126/scitranslmed.3004685

Saper CB, Fuller PM, Pedersen NP, Lu J, Scammell TE (2010) Sleep state switching. Neuron 68:1023-1042. https://doi.org/10.1016/j.neuron. 2010.11.032

Scammell TE, Arrigoni E, Lipton JO (2017) Neural circuitry of wakefulness and sleep. Neuron 93:747-765. https://doi.org/10.1016/j. neuron.2017.01.014
Timby E, Backstrom T, Nyberg S, Stenlund H, Wihlback AC, Bixo M (2016) Women with premenstrual dysphoric disorder have altered sensitivity to allopregnanolone over the menstrual cycle compared to controls - a pilot study. Psychopharmacology 233:2109-2117. https://doi.org/10.1007/s00213-016-4258-1

Timby E, Balgård M, Nyberg S, Spigset $\mathrm{O}$, Andersson A, PorankiewiczAsplund J, Purdy RH, Zhu D, Bäckström T, Poromaa IS (2006) Pharmacokinetic and behavioral effects of allopregnanolone in healthy women. Psychopharmacology 186:414-424. https://doi. org/10.1007/s00213-005-0148-7

Trotti LM, Saini P, Koola C, LaBarbera V, Bliwise DL, Rye DB (2016) Flumazenil for the treatment of refractory hypersomnolence: clinical experience with 153 patients. J Clin Sleep Med 12:1389-1394. https://doi.org/10.5664/jcsm.6196

van Broekhoven F, Backstrom T, van Luijtelaar G, Buitelaar JK, Smits P, Verkes RJ (2007) Effects of allopregnanolone on sedation in men, and in women on oral contraceptives. Psychoneuroendocrinology 32:555-564. https://doi.org/10.1016/j.psyneuen.2007.03.009

Wang MD, Wahlstrom G, Gee KW, Backstrom T (1995) Potency of lipid and protein formulation of 5 alpha-pregnanolone at induction of anaesthesia and the corresponding regional brain distribution. $\mathrm{Br} \mathrm{J}$ Anaesth 74:553-557

Venner A, Anaclet C, Broadhurst RY, Saper CB, Fuller PM (2016) A novel population of wake-promoting GABAergic neurons in the ventral lateral hypothalamus. Curr Biol 26:2137-2143. https://doi. org/10.1016/j.cub.2016.05.078

Wilson SJ, Glue P, Ball D, Nutt DJ (1993) Saccadic eye movement parameters in normal subjects. Electroencephalogr Clin Neurophysiol 86:69-74

Vilstrup H, Amodio P, Bajaj J, Cordoba J, Ferenci P, Mullen KD, Weissenborn K, Wong P (2014) Hepatic encephalopathy in chronic liver disease: 2014 practice guideline by the American Association for the Study of Liver Diseases and the European Association for the Study of the Liver. Hepatology 60:715-735. https://doi.org/10. 1002/hep. 27210

Winsky-Sommerer R (2009) Role of GABAA receptors in the physiology and pharmacology of sleep. Eur J Neurosci 29:1779-1794. https://doi.org/10.1111/j.1460-9568.2009.06716.x

Volgin DV, Lu JW, Stettner GM, Mann GL, Ross RJ, Morrison AR, Kubin L (2014) Time- and behavioral state-dependent changes in posterior hypothalamic GABAA receptors contribute to the regulation of sleep. PLoS One 9:e86545. https://doi.org/10.1371/journal. pone. 0086545 